Chapter 10

\title{
A Metabolomics Approach to Metabolic Diseases
}

\author{
Luis Aldámiz-Echevarría, Fernando Andrade, \\ Marta Llarena and Domingo González-Lamuño
}

Additional information is available at the end of the chapter

http://dx.doi.org/10.5772/65469

\begin{abstract}
Metabolomics, defined as the comprehensive analysis of compounds in a biological specimen, is an emerging technology that helps several pathologies to inform about new biomarkers. Metabolic diseases comprise a group of rare conditions that in total represent an important health problem. Historically, small numbers of metabolites have been used to diagnose complex metabolic diseases such as diabetes or metabolic diseases. Metabolomic methodology, due to the evolution of clinical chemistry technologies, could detect thousands of organic compounds. In this way, metabolomic approach gives information of metabolic pathways describing physiopathology that underlies disease, including the possibility of discovery of new markers that could be used to diagnose or check the efficacy of the treatments. Diabetes, classic inborn error of metabolism as methylmalonic aciduria, lysosomal diseases and rare optic neuropathy affecting adults are discussed in this chapter.
\end{abstract}

Keywords: metabolic diseases, diabetes, optic neuropathy, lysosomal diseases

\section{Introduction}

Rare metabolic diseases comprise a group of more than 7000 conditions that are particularly rare, but in total, they represent an important health problem. There is no single, internationally accepted definition of a rare metabolic disease; currently, it is a syndrome that occurs in one child per 200,000 births. In Europe, they are supposed to affect $7 \%$ of the population. Nowadays, as less than 500 metabolic diseases have available and effective treatments, new therapeutic solutions should be developed.

New approach and initiatives are necessary to advance research for patients suffering from an inborn error of metabolism because governments pay little attention due to their costs and 
low incidences. Suitable and quick diagnosis is basic to the patients, even if there is no treatment, since it reduces the overdiagnosis, followed by suitable care, can improve quality of life for these patients.

The inherited metabolic diseases are biochemical defects diagnosed routinely by neonatal screening programmes. This successful screening methodology continues to broaden and improve, and new biomarkers are added depending on the country.

Metabolomics is an "omics" science focused on dynamic biology ruled by organic metabolites. A metabolome is defined as the group of metabolites detected and playing in the same metabolic pathway in the normal functioning of the cell. Metabolomics complements other analytical sciences as proteomics or genomics. Physiological changes that are consequences of a special gene expression are characterised by a variation in the metabolic compounds. Thus, the metabolome is more sensitive than the transcriptome or the proteome. A small variation in protein expression can have a significant effect on the activity of the metabolic pathway and the concentration of the relevant metabolites.

In summary, the workflow for metabolomics applied to metabolic diseases starts with model design or patients selection (inclusion/exclusion criteria), sample preparation and separationdetection of metabolites by chromatography and mass spectrometry, respectively. Then, the obtained information has to be aligned and identified in order to carry out the statistics. The last step in this methodology is to obtain useful biomarkers for each inborn error of metabolism (Figure 1).

The aim of this chapter is to show the utility of metabolomics, applying to diabetes and inborn error of metabolism. The latter ones traditionally with difficult diagnosis and uncertain treatment are due to their low incidence. However, the metabolomic approach could give these kinds of diseases a tool to discover new and effective biomarkers.

\subsection{Diabetes}

Diabetes, caused by several interactions between genetic and environmental factors, is a frequent disorder related to mutations in several genes, with each individual gene accounting for $1 \%$ of disease risk. In case of type 2 diabetes, dysfunction of multiple organ systems, including impaired insulin action in the muscle and adipose tissue, defective hepatic glucose production and insulin resistance are caused by loss of beta-cell mass and function. The difficult challenge for the understanding of the molecular pathways is evident, but progress in this area may be aided by the recent advent of technologies for metabolomic approach including nuclear magnetic resonance or mass spectrometry.

Metabolomic could provide some advantages over other "omics" technologies in diabetes research: (1) The amount of metabolites or small molecules found and identified is less than the number of genes or proteins. So, the pathway interpretation should be easier and effective, as the results from genomics and proteomics. (2) Metabolomics is a tool for describing mechanisms of action and reverse effects of several treatments. However, metabolomics can be a science with limitations for technology misuse or data overinterpretation. 


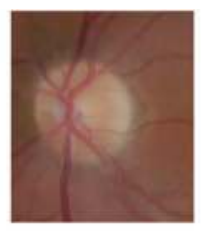

Metabolic disease patients
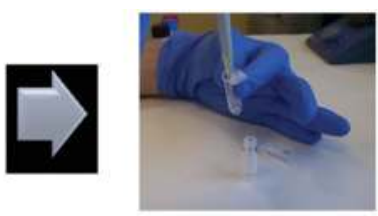

Sample pretreatment
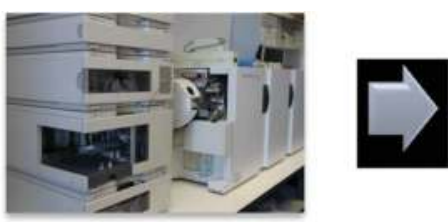

Separation and detection

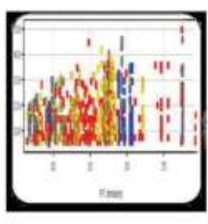

Alignment

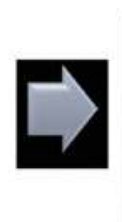

Identification

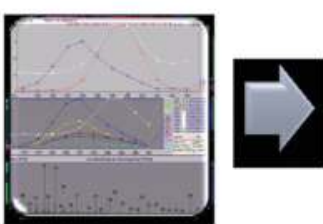

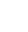
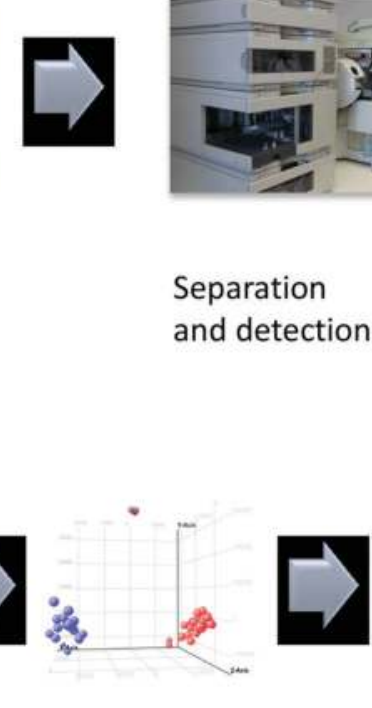

Stadistics

(n)


As some diabetic patients could suffer from alterations in energy metabolism, the study of creatine levels could be imperative to indicate a possible deficit of creatine to muscles or the brain. This creatine becomes creatine phosphate by a process of phosphorylation, leading to the genesis of ATP, essential in tissues with high-energy consumption, such as muscles and the brain through a process of dephosphorylation. An alteration of this metabolic pathway would lead to increase guanidinoacetate and a deficit in the production of creatine and, therefore, phosphocreatine.

\section{Carnitine}

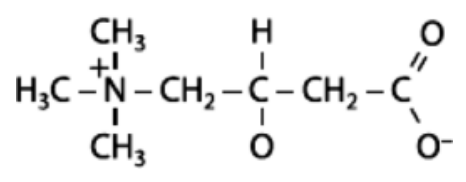

Figure 2. Molecular structure of carnitine, extracted from plasma by removing proteins.

In the absence of specific metabolic biomarkers of evolution and efficacy to treatment in diabetes, it should be performed a metabolic profile based on the profile of amino acids, which have been related to the methylation cycle and dementia (Met, Cys, Tau) in the diabetes.

L-Arginine, the main substrate of the endothelial nitric oxide synthase (NOS), is oxidised to L-citrulline and nitric oxide. Asymmetric dimethylarginine (ADMA) is derived from the proteolysis of proteins and acts as a competitive inhibitor of nitric oxide synthase, which is related to blood pressure, glucose intolerance, the thickness of the intima-media carotid, etc., making it a cardiovascular marker. It has been observed that the endothelial NO production is reduced in diabetic patients at high cardiovascular risk partly due to the limitation in the synthesis of arginine substrate and, secondly, because the ADMA, its inhibitor, is increased. So, it is proposed that the ADMA acts as a marker of cardiovascular risk in diabetic patients [5].

Epigenetics may contribute to metabolic study of diabetes through the global status of DNA methylation, which has been vaguely studied in this disease. Such mechanism methylation may play a role in the aetiology of monogenic diabetes. We propose the quantification of 5-methylcytosine (5-mC) in order to study changes in the methylation of DNA/RNA or its possible degradation during the treatment.

\subsection{Methylmalonic aciduria}

Methylmalonic aciduria (MMA) is an inherited disorder involving certain amino acid and fatty acid metabolism. Levels of methylmalonic acid are increased mainly in urine. They are caused by an enzymatic deficiency of methylmalonyl-CoA mutase (MUT) in the mitochondria or by a defect in the uptake, transport or synthesis of cobalamin, the cofactor of MUT. The management of this organic aciduria is based on a low-protein diet. MMA patient could suffer 
from renal failure as one of their critical problems, not common in other organic acidurias. However, the cause of renal failure and cardiovascular complications depends on several pathways. So, it is thought that nitric oxide formation could be affected causing endothelial dysfunction. Related to nitric oxide, arginine-asymmetric dimethylarginine (ADMA) pathway, where ADMA acts as an inhibitor for nitric oxide synthase, should be described in these patients. ADMA levels are associated with an increase in cardiovascular events and chronic renal disease [6]. In addition, levels of the isomer symmetric dimethylarginine (SDMA) as a renal biomarker were also elevated in patients with chronic kidney disease.

Metabolic approach to evaluate the cardiovascular biomarker status should be recommended to improve the management for these patients. The objectives of this approach are also the identification and quantification of biomarkers of renal injury and inflammation such as interleukins, tumour necrosis factor alpha (TNF $\alpha$ ) and transforming growth factor beta (TGF $\beta$ ), in patients who are at different stages of kidney damage. The definition of biomarkers for early renal impairment in MMA patient could assess changes in these parameters during the kidney damage progression.

The comparison of MMA subgroups at different stages of kidney damage would allow the identification of those markers of inflammation and tissue fibrosis involved in tubulointerstitial nephritis or primary glomerular injury and will establish a chronology on the appearance of altered levels of biomarkers for the development of kidney damage. Finally, we believe that greater understanding of the role of these molecules and inflammatory processes in the context of associated nephropathy by means of metabolomics is vital to facilitate the development of novel targets and therapeutic nephroprotective strategies. The use of anti-inflammatory molecules or antioxidants can be applied clinically successfully.

\subsection{Non-arteritic anterior ischemic optic neuropathy (NAION)}

The cause for optic neuropathy affecting elderly patients could be multifactorial and difficult to diagnose in case of non-arteritic anterior ischemic optic neuropathy (NAION). Few information has been published about metabolic pathways involved in this rare disease. So, the purpose of metabolomics is to suggest effective biomarkers and to describe metabolic fingerprinting.

Samples of patients and controls can be fingerprinted with liquid chromatography coupled to high-sensitive mass spectrometry, such as quadrupole time of flight (QTOF). According to Figure 1, data should be filtered, aligned and statistically analysed before identifying new compounds. These biomarkers were found to be significant in class separation and could be later confirmed by obtaining the characteristic MS/MS spectra and online databases, as Metlin or Human Metabolome Database. NAION patients presented differences in the phospholipid profile in comparison with controls, such as lower levels of lysoPCs.

LysoPCs and lysoPEs are products or metabolites of PCs and PEs (Figure 3), respectively, which are structural components of cell membranes. The structure of lysoPCs is a choline polar group linked to fatty acyls that differ in chain length, position and degree of 

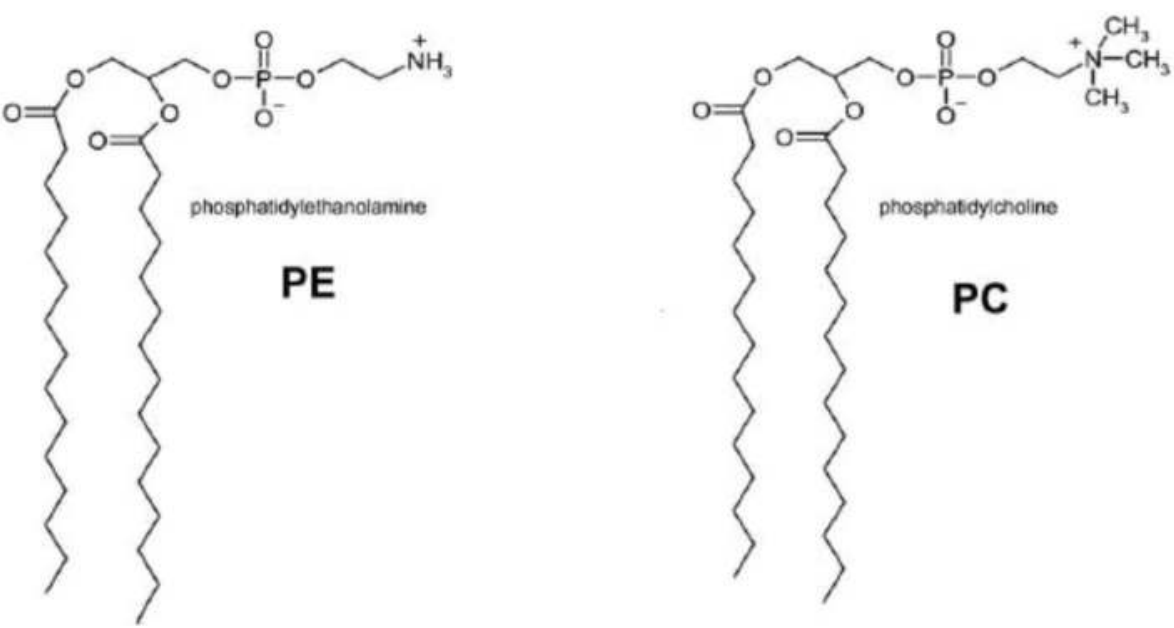

Figure 3. Molecular structure for phosphatidylcholine (PC) and phosphatidylethanolamine (PE), as the precursors or LysoPC and LysoPE.

saturation. These compounds were extracted from blood samples by centrifugation, so protein precipitation was performed by adding $300 \mu \mathrm{L}$ of cool methanol:ethanol (1:1) to the plasma. They were vortexed during $1 \mathrm{~min}$ and stored at $-20{ }^{\circ} \mathrm{C}$ for $10 \mathrm{~min}$. After centrifugation, the supernatant can be injected in MS equipment. Increased levels of lysoPEs and reduced levels of lysoPCs can be a consequence of an altered choline phospholipid metabolism. The whole profile of lysoPCs has been published as a biomarker in other diseases, though the type of the lysoPCs was not taken into account [7]. As lysoPC profile is a biomarker for other diseases, variations in some lysoPC isomers may not be enough in order to identify a specific disease. For this reason, individual lysoPC levels produced by a pathology should be further investigated. As it has been researched by our group, lysoPC profiling of plasma shows a characteristic profile in NAION patients: higher levels of lysoPEs and lower levels of lysoPCs. As PCs are methylated products of PEs, the hypothesis for this result revolves around the poor methylation capacity in optic nerve cells. The characteristic profile of lysoPCs and lysoPEs also shows that the activity of phospholipases (PL) and lysophospholipases (LPL) could be increased [8].

NAION patients showed high levels of L-palmitoylcarnitine, which has the highest weight in the Partial Least Squares Discriminant Analysis (PLS-DA) prediction model (Figure 4). Nevertheless, these levels are not clearly related to the physiopathology of the disease because the uptake of acetylcarnitines by the mitochondria is a reversible process that is used to undergo mitochondrial $\beta$-oxidation of long-chain fatty acids and to transport acyl-coenzyme A from mitochondria to cytosol. So, more studies are necessary to confirm a disturbance in $\beta$-oxidation of fatty acid. However, these high levels of L-palmitoylcarnitine have been involved in the pathology of ischemia, acting as an inhibitor of cardiac $\mathrm{Na}$,K-ATPase. Because of its amphipathic skill, palmitoylcarnitine can induce alterations in membrane fluidity and 


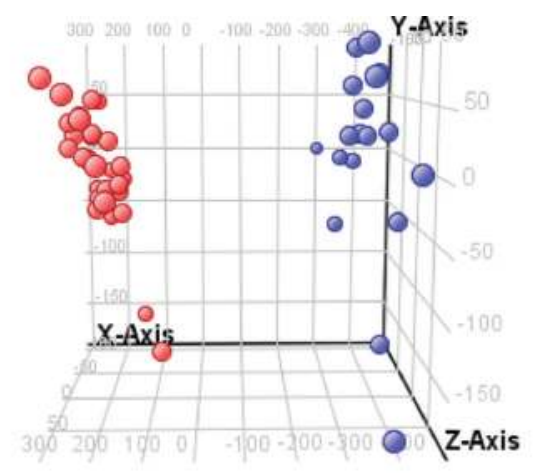

$x$-Axis

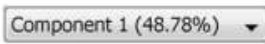

Y-Axis

Component $2(9.73 \%)$

Z-Axis

Component $3(5.84 \%)$

Figure 4. Partial Least Squares Discriminant Analysis (PLS-DA) scores plot of plasma metabolic profiles obtained from NAION patients and controls.

surface during apoptosis in NAION patients [9]. In this case, the membrane disturbance takes part in high-energy uptake cells of the optic nerve provoking severe blindness. This discussion could signify that carnitine as a supplement could increase the in vivo regeneration of optic nerve cells after NAION event. Furthermore, due to identified profile by metabolomics and NAION physiopathology as central nervous system problem, it seems to be a stronger relationship between NAION disease and other ischemic processes, such as myocardial ischemia or ictus, than with neuropathological or ophthalmological syndromes.

\subsection{Lysosomal diseases}

The lysosomal diseases are another group of inherited metabolic disorders characterised by mutations in genes encoding lysosomal enzymes and proteins. The enzyme deficiencies cause impaired intracellular turnover and build-up of complex molecules including sphingolipids, glycosaminoglycans and glycoproteins. These metabolites are established by metabolic approach as biomarkers. The pathology of lysosomal diseases is typically characterised by intra-lysosomal storage of a variety of substrates in multiple tissues and organs. Thus, the phenotypes of these disorders are complex and characterised by the variable association of visceral, skeletal and neurological manifestations.

The advancement in the diagnosis and treatment of lysosomal diseases has been largely stimulated by the improved knowledge on their molecular bases and pathophysiology. Metabolomics has a major role in this further impulse to the availability of technologies allowing faster diagnosis. In the case of Fabry disease, caused by deficiency of $\alpha$-galactosidase $\mathrm{A}$, the glycosphingolipid accumulation in plasma and urine is used as biomarker. Two specific biomarkers have been identified and quantified in plasma and urine: globotriaosylceramide (Gb3) and globotriaosylsphingosine (lyso-Gb3). The search continues for biomarkers that might be reliable indicators of disease severity and response to treatment. 
Several metabolomic studies have been carried out for lysosomal diseases, such as Fabry [10] and Niemann-Pick [11] syndromes, to establish efficient biomarkers. For other lysosomal diseases like mucopolysaccharidoses, these studies could be useful to identify the increase of a specific glycosaminoglycan in urine, such as dermatan sulphate, chondroitin sulphate, heparan sulphate and keratan sulphate [12].

\section{Future progress}

Nowadays, the metabolomics is continuously improving its methodology to obtain new biomarkers quickly by means of advances in mass spectrometry and statistics software. So, it is clear that little-studied rare diseases, with no defined biomarkers, will be studied through this methodology. However, what is going to be an outstanding breakthrough is the combination of the information obtained by metabolomics, proteomics and genomics. Sometimes, these "omics" sciences are studied separately, but all information should be taken into account together in the future to understand the connexion of all information in the whole metabolic pathway.

\section{Conclusions}

It is evident that there is not a single perfect tool for global metabolic profiling. However, metabolomics, which combines sensitive analytical techniques with multivariate analysis, seems to be a good approach to identify biomarkers in diabetes and metabolic rare diseases, such as methylmalonic acidemia or lysosomal diseases.

\section{Acknowledgements}

Research and results in this manuscript were financed by the BioCruces and Carlos III Health Research Institutes.

\section{Appendices and nomenclatures}

ADMA Asymmetric dimethylarginine

HMDB Human Metabolome Database

LysoPC Lysophosphatidylcholine

LysoPE Lysophosphatidylethanolamine

MMA Methylmalonic acidurias 
NAION Non-arteritic anterior ischemic optic neuropathy

PC Phosphatidylcholine

PE Phosphatidylethanolamine

PLS-DA Partial least squares discriminant analysis

SDMA Symmetric dimethylarginine

\section{Author details}

Luis Aldámiz-Echevarría ${ }^{1,}$, Fernando Andrade ${ }^{1}$, Marta Llarena $^{1}$ and Domingo GonzálezLamuño ${ }^{2}$

*Address all correspondence to: luisjose.aldamiz-echevarazuara@osakidetza.eus

1 Division of Metabolism, BioCruces Health Research Institute, Centre for Biomedical Research on Rare Diseases (CIBER-ER), Barakaldo, Bizkaia, Spain

2 Department of Nephrology and Metabolism, Marqués de Valdecilla University Hospital, Santander, Spain

\section{References}

[1] Bain JR, Stevens RD, Wenner BR, Ilkayeva O, Muoio DM, Newgard CB. Metabolomics applied to diabetes research: moving from information to knowledge. Diabetes 2009;58:2429-2443. doi: 10.2337/db09-0580

[2] Wang-Sattler R, Yu Z, Herder C, Messias AC, Floegel A, He Y, et al. Novel biomarkers for pre-diabetes identified by metabolomics. Mol Syst Biol 2012;8:615. doi: 10.1038/ msb.2012.43

[3] Adams SH, Hoppel CL, Lok KH, Zhao L, Wong SW, Minkler PE, Hwang DH, Newman JW, Garvey WT. Plasma acylcarnitine profiles suggest incomplete long-chain fatty acid beta-oxidation and altered tricarboxylic acid cycle activity in type 2 diabetic AfricanAmerican women. J Nutr 2009;139:1073-1081. doi: 10.3945/jn.108.103754

[4] Mamoulakis D, Galanakis E, Dionyssopoulou E, Evangeliou A, Sbyrakis S. Carnitine deficiency in children and adolescents with type 1 diabetes. J Diabetes Complications 2004;18:271-274. doi: 10.1016/S1056-8727(03)00091-6

[5] Tarnow L, Hovind P, Teerlink T, Stehouwer CD, Parving HH. Elevated plasma asymmetric dimethylarginine as a marker of cardiovascular morbidity in early diabetic nephropathy in type 1 diabetes. Diabetes Care 2004;27(3):765-769. doi: 10.2337/ diacare.27.3.765 
[6] Lu TM, Chung MY, Lin CC, Hsu CP, Lin SJ. Asymmetric dimethylarginine and clinical outcomes in chronic kidney disease. Clin J Am Soc Nephrol 2011;6:1566-1572. doi: 10.2215/CJN.08490910

[7] Dong J, Cai X, Zhao L, Xue X, Zou L, Zhang X, Liang X. Lysophosphatidylcholine profiling of plasma: discrimination of isomers and discovery of lung cancer biomarkers. Metabolomics 2010:6;478-488. doi: 10.1007/s11306-010-0215-x

[8] Ciborowski M, Rupérez FJ, Martinez-Alcázar MP, Angulo S, Radziwon P, Olszanski R, Kioczko J, Barbas C. Metabolomic approach with LC-MS reveals significant effect of pressure on Diver's plasma. J Proteome Res 2010;9:4131-4137. doi: 10.1021/pr100331j

[9] Nalecz KA, Miecz D, Berezowski V, Cecchelli R. Carnitine: transport and physiological functions in the brain. Mol Aspects Med 2004;25:551-567. doi: 10.1016/j.mam.2004.06.001

[10] Boutin M, Auray-Blais C. Metabolomic discovery of novel urinary galabiosylceramide analogs as Fabry disease biomarkers. J Am Soc Mass Spectrom 2015;26(3):499510. doi: 10.1007/s13361-014-1060-3

[11] Maekawa M, Shimada M, Ohno K, Togawa M, Nittono H, Iida T, Hofmann AF, Goto J, Yamaguchi H, Mano N. Focused metabolomics using liquid chromatography/ electrospray ionization tandem mass spectrometry for analysis of urinary conjugated cholesterol metabolites from patients with Niemann-Pick disease type $C$ and 3ß-hydroxysteroid dehydrogenase deficiency. Ann Clin Biochem 2015;52:576-587. doi: $10.1177 / 0004563214568871$

[12] Staples GA, Zaia J. Analysis of glycosaminoglycans using mass spectrometry. Curr Proteomics 2011;8:325-336. doi: 10.2174/157016411798220871 